\title{
Manganese Nanoparticles Control Salinity-Modulated Molecular Responses in Capsicum annuum L. through Priming: A Sustainable Approach for Agriculture
}

Yuqing Yeac, Keni Cota-Ruiz ${ }^{\text {ac }}$, José A. Hernández-Viezcas ${ }^{a c}$, Carolina Valdés ${ }^{a}$, Illya A. MedinaVelo $^{\text {d }}$, Reagan S. Turley ${ }^{a}$, Jose R. Peralta-Videa ${ }^{\text {abc }}$, and Jorge L. Gardea-Torresdey ${ }^{\text {abc* }}$

aDepartment of Chemistry and Biochemistry, The University of Texas at El Paso, 500 West University Avenue, E1 Paso, TX 79968, USA

bEnvironmental Science and Engineering Ph.D. program, The University of Texas at El Paso, 500 West University Avenue, El Paso, TX 79968, USA.

cUC Center for Environmental Implications of Nanotechnology (UC CEIN), The University of Texas at El Paso, 500 West University Avenue, El Paso, TX 79968, USA.

dDepartment of Natural Sciences, Western New Mexico University; 1000 W College Ave., Silver City, NM 88062, United States.

*Corresponding author: jgardea@utep.edu (J.L. Gardea) Phone: 915747 5359, Fax: 915747 5748

Number of pages: 12

Number of Figures: 3

Number of Tables: 8 


\section{Supporting information}

Table S1. Micro/macro nutrient content in roots of 14 day old $C$. annuum seedlings 


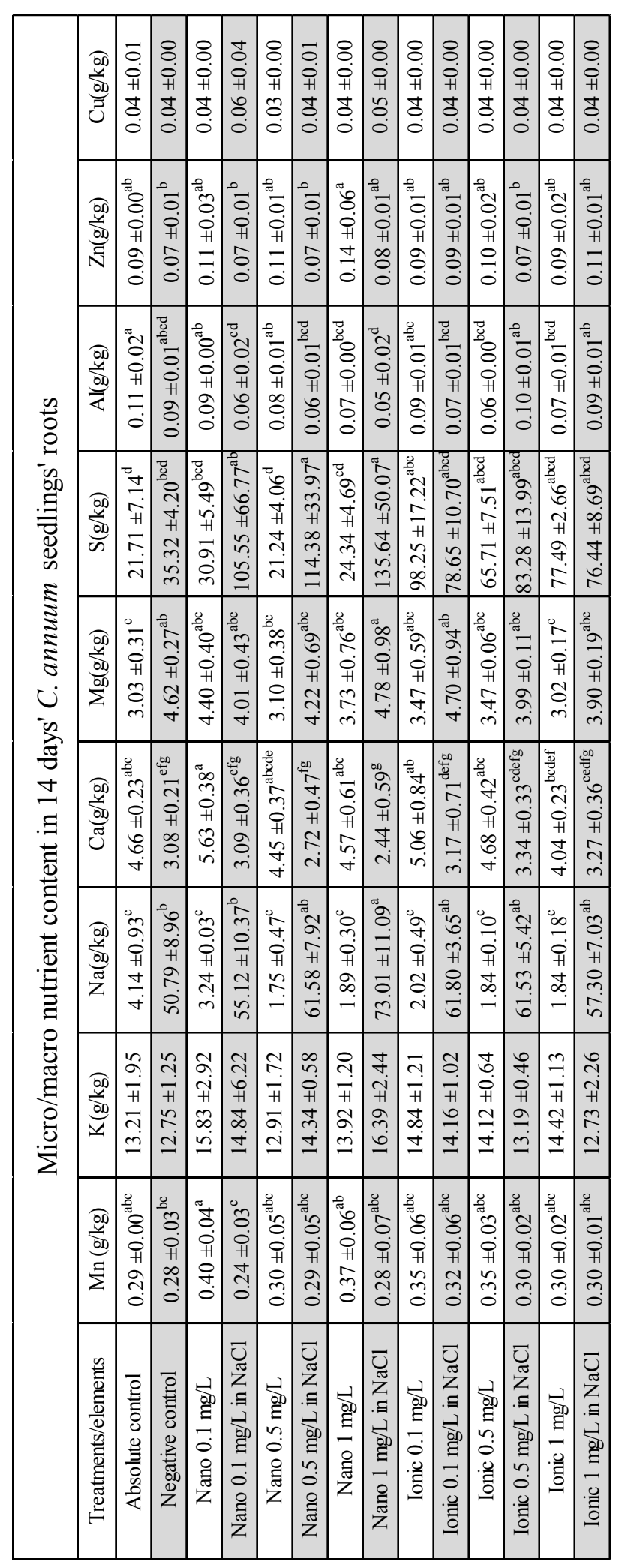

Table S2. Micro/macro nutrient content in shoots of 14 day old $C$. annuum seedlings 


\begin{tabular}{|c|c|c|c|c|c|c|c|c|c|c|c|c|c|c|c|}
\hline \multirow{10}{*}{ 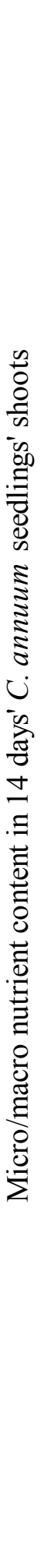 } & है & $\mid \begin{array}{l}0 \\
\dot{0} \\
+1 \\
\tilde{1} \\
0 \\
0\end{array}$ & $\begin{array}{l}8 \\
0 \\
+ \\
+1 \\
m \\
0 \\
0\end{array}$ & $\begin{array}{l}0 \\
0 \\
+1 \\
\tilde{y} \\
0\end{array}$ & $\left|\begin{array}{l}-1 \\
0 \\
0 \\
+1 \\
0 \\
0 \\
0\end{array}\right|$ & $\begin{array}{l}8 \\
0 \\
0 \\
+1 \\
0 \\
0 \\
0\end{array}$ & $\begin{array}{l}8 \\
0 \\
0 \\
+1 \\
\tilde{o} \\
0 \\
0\end{array}$ & $\left|\begin{array}{l}0 \\
0 \\
+1 \\
0 \\
0 \\
0\end{array}\right|$ & $\mid \begin{array}{c}-1 \\
0 \\
0 \\
+1 \\
+1 \\
0 \\
0 \\
0\end{array}$ & $\left|\begin{array}{l}0 \\
0 \\
\dot{i} \\
+1 \\
\ddot{0} \\
0 \\
0\end{array}\right|$ & $\begin{array}{l}8 \\
0 \\
0 \\
+1 \\
0 \\
0 \\
0\end{array}$ & $\mid$\begin{tabular}{l|}
8 \\
0 \\
0 \\
+1 \\
$\tilde{o}$ \\
0 \\
0
\end{tabular} & $\begin{array}{l}8 \\
0 \\
+1 \\
\tilde{n} \\
0 \\
0\end{array}$ & $\mid \begin{array}{l}0 \\
0 \\
0 \\
+1 \\
\tilde{y} \\
0 \\
0\end{array}$ & $\begin{array}{l}0 \\
\dot{0} \\
+1 \\
\tilde{+} \\
0 \\
\dot{0}\end{array}$ \\
\hline & $\begin{array}{l}000 \\
\frac{100}{0}\end{array}$ & $\mid \begin{array}{l}0 \\
\dot{0} \\
+1 \\
0 \\
0 \\
0\end{array}$ & $\begin{array}{l}8 \\
0 \\
+1 \\
+1 \\
u \\
0 \\
0\end{array}$ & $\begin{array}{l}0 \\
0 \\
+1 \\
0 \\
0 \\
0\end{array}$ & $\left|\begin{array}{l}\tilde{O} \\
0 \\
+1 \\
+1 \\
\varrho \\
0 \\
0\end{array}\right|$ & $\begin{array}{l}-1 \\
0 \\
+1 \\
+1 \\
0 \\
0\end{array}$ & $\begin{array}{l}-1 \\
0 \\
0 \\
+1 \\
0 \\
0 \\
0\end{array}$ & $\begin{array}{l}8 \\
0 \\
+1 \\
+1 \\
\varnothing \\
0\end{array}$ & $\left|\begin{array}{l}8 \\
\dot{0} \\
+1 \\
0 \\
0 \\
0\end{array}\right|$ & $\left|\begin{array}{l}-1 \\
0 \\
0 \\
+1 \\
o \\
0 \\
0\end{array}\right|$ & $\begin{array}{l}8 \\
0 \\
+1 \\
+1 \\
20 \\
0 \\
0\end{array}$ & $\left|\begin{array}{l}-1 \\
0 \\
0 \\
+1 \\
0 \\
0 \\
0\end{array}\right|$ & $\begin{array}{l}-1 \\
0 \\
+1 \\
+1 \\
\stackrel{+}{0} \\
0\end{array}$ & $\left|\begin{array}{l}8 \\
0 \\
+1 \\
+1 \\
\varnothing \\
0\end{array}\right|$ & $\mid \begin{array}{l}8 \\
\dot{0} \\
+1 \\
+1 \\
0 \\
0 \\
0\end{array}$ \\
\hline & & \begin{tabular}{|c|} 
\\
0 \\
0 \\
0 \\
+1 \\
0 \\
0 \\
0 \\
0
\end{tabular} & $\begin{array}{ll}0 & 0 \\
0 \\
0 \\
0 \\
+1 \\
+1 \\
0 \\
0 \\
0\end{array}$ & 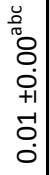 & $\mid$\begin{tabular}{|c|}
0 \\
0 \\
0 \\
0 \\
0 \\
+1 \\
+1 \\
-0 \\
0 \\
0 \\
\end{tabular} & \begin{tabular}{|l|}
0 \\
0 \\
0 \\
0 \\
0 \\
+1 \\
+1 \\
0 \\
0 \\
0
\end{tabular} & \begin{tabular}{|c|c}
0 \\
0 \\
0 \\
0 \\
0 \\
+1 \\
+1 \\
-1 \\
0 \\
0
\end{tabular} & $\begin{array}{c}0 \\
0 \\
0+1 \\
+1 \\
0 \\
0 \\
.\end{array}$ & \begin{tabular}{|l|} 
\\
8 \\
0 \\
0 \\
+1 \\
-1 \\
0 \\
0
\end{tabular} & $\mid \begin{array}{l}0 \\
0 \\
\sigma \\
0 \\
0 \\
+1 \\
+1 \\
0 \\
0\end{array}$ & $\begin{array}{l}0 \\
\stackrel{0}{0} \\
8 \\
0 \\
+1 \\
+1 \\
0 \\
0 \\
0\end{array}$ & $\mid \begin{array}{c}0 \\
0 \\
0 \\
0 \\
0+1 \\
0 \\
0 \\
0 \\
0\end{array}$ & $\begin{array}{l}0 \\
0 \\
0 \\
0 \\
+1 \\
-1 \\
-0 \\
0\end{array}$ & $\mid \begin{array}{c}0 \\
0 \\
0 \\
+1 \\
+1 \\
-1 \\
0 \\
0\end{array}$ & $\left|\begin{array}{c}0 \\
0 \\
0 \\
0+1 \\
+1 \\
0 \\
0 \\
0\end{array}\right|$ \\
\hline & 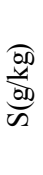 & 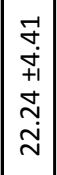 & $\begin{array}{c}g \\
\stackrel{2}{+} \\
\dot{+} \\
+1 \\
\vec{m} \\
\vec{m}\end{array}$ & $\begin{array}{l}\stackrel{\overbrace{}}{\sim} \\
\stackrel{+}{+} \\
+1 \\
\stackrel{\sim}{n} \\
\stackrel{2}{\sim} \\
\end{array}$ & 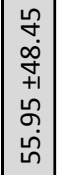 & 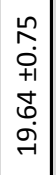 & $\begin{array}{l}\tilde{N} \\
\sim \\
\dot{+} \\
+1 \\
\infty \\
\ddot{\sim} \\
\dot{\sim}\end{array}$ & $\begin{array}{c}\overrightarrow{-} \\
\infty \\
i \\
+1 \\
0 \\
m \\
\infty \\
\sim \\
\sim\end{array}$ & 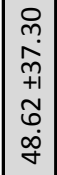 & 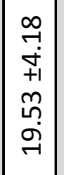 & 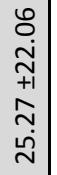 & $\left|\begin{array}{c}\hat{0} \\
+ \\
++1 \\
0 \\
\leftrightarrow \\
- \\
-1\end{array}\right|$ & 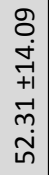 & 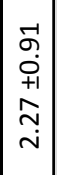 & $\begin{array}{c}\infty \\
\stackrel{\infty}{ } \\
\dot{p} \\
+1 \\
\tilde{n} \\
\tilde{r} \\
\dot{\gamma}\end{array}$ \\
\hline & 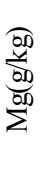 & 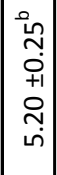 & 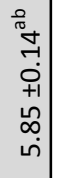 & 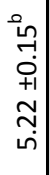 & $\mid \begin{array}{l}0 \\
0 \\
0 \\
0 \\
+1 \\
\hat{n} \\
0 \\
0\end{array}$ & 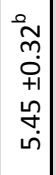 & 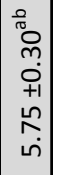 & 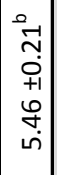 & \begin{tabular}{|c|c|}
0 \\
$\tilde{0}$ \\
$\tilde{m}$ \\
0 \\
0 \\
+1 \\
0 \\
0 \\
$\infty$ \\
$\dot{\omega n}$
\end{tabular} & 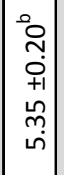 & 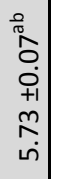 & 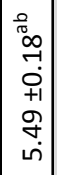 & 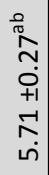 & 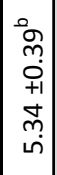 & 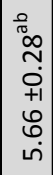 \\
\hline & 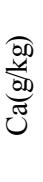 & $\mid \begin{array}{c}0 \\
0 \\
0 \\
0 \\
+1 \\
-1 \\
0 \\
-i\end{array}$ & 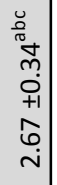 & $\begin{array}{l}\breve{g} \\
0 \\
0 \\
+1 \\
+ \\
\stackrel{-}{-} \\
-i\end{array}$ & 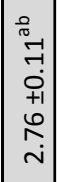 & $\begin{array}{l}0 \\
0 \\
0 \\
0 \\
0 \\
+1 \\
0 \\
0 \\
0 \\
-i\end{array}$ & 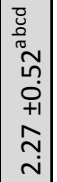 & 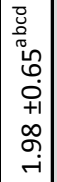 & 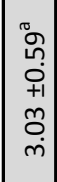 & $\left|\begin{array}{c}0 \\
0 \\
0 \\
0 \\
+1 \\
0 \\
0 \\
\\
\rightarrow i\end{array}\right|$ & 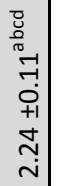 & 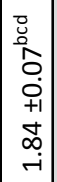 & 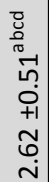 & $\mid \begin{array}{c}0 \\
\text { in } \\
0 \\
+0 \\
+1 \\
0 \\
0 \\
-1 \\
-i\end{array}$ & 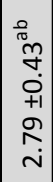 \\
\hline & 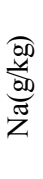 & $\left|\begin{array}{l}0 \\
0 \\
0 \\
+1 \\
\tilde{n} \\
0 \\
0\end{array}\right|$ & 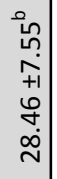 & 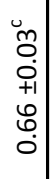 & $\mid$ & $\begin{array}{l}0 \\
0 \\
\dot{0} \\
+1 \\
\dot{+1} \\
\dot{0}\end{array}$ & 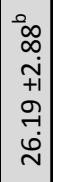 & 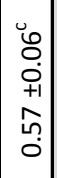 & 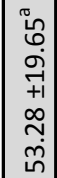 & 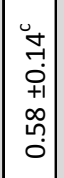 & 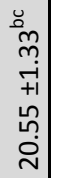 & 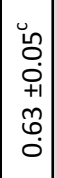 & $\begin{array}{l}0 \\
\tilde{n} \\
0 \\
+1 \\
+1 \\
0 \\
\dot{\omega} \\
\dot{v}\end{array}$ & 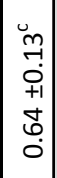 & 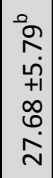 \\
\hline & 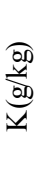 & 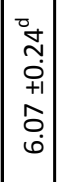 & 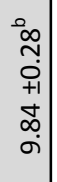 & 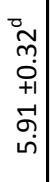 & 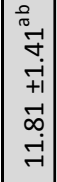 & 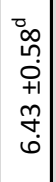 & 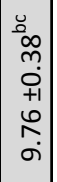 & 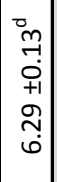 & 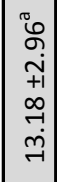 & $\left|\begin{array}{c}0 \\
0 \\
0 \\
0 \\
+1 \\
+1 \\
గ 1 \\
6\end{array}\right|$ & 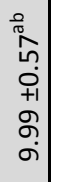 & 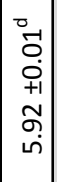 & 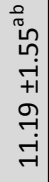 & 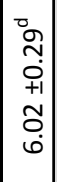 & 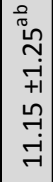 \\
\hline & 3 & 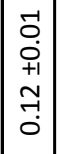 & $\begin{array}{c}-1 \\
0 \\
0 \\
+1 \\
0 \\
0 \\
0\end{array}$ & $\begin{array}{c}8 \\
\stackrel{+}{+} \\
+1 \\
m \\
\dddot{\sim} \\
0\end{array}$ & $\mid \begin{array}{c}\tilde{O} \\
0 \\
0 \\
+1 \\
0 \\
0 \\
0 \\
0\end{array}$ & $\begin{array}{c}-1 \\
0 \\
0 \\
+1 \\
+ \\
-1 \\
0\end{array}$ & 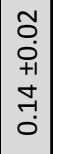 & $\begin{array}{c}-1 \\
0 \\
0 \\
+1 \\
+ \\
-1 \\
0\end{array}$ & $\mid \begin{array}{c}-1 \\
0 \\
0 \\
+1 \\
\infty \\
0 \\
0 \\
0\end{array}$ & $\begin{array}{c}\overrightarrow{-} \\
0 \\
\dot{+1} \\
+ \\
\overrightarrow{0} \\
\stackrel{0}{0}\end{array}$ & $\begin{array}{c}-1 \\
0 \\
0 \\
+1 \\
-1 \\
-1 \\
0\end{array}$ & \begin{tabular}{|c|}
8 \\
0 \\
0 \\
+1 \\
$n$ \\
\\
0
\end{tabular} & $\begin{array}{l}n \\
0 \\
0 \\
+1 \\
+ \\
\stackrel{1}{0} \\
0\end{array}$ & $\mid \begin{array}{c}-1 \\
0 \\
0 \\
+1 \\
+1 \\
-1 \\
0\end{array}$ & $\mid \begin{array}{l}0 \\
0 \\
0 \\
+1 \\
0 \\
0 \\
-1 \\
0\end{array}$ \\
\hline & d & 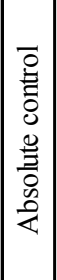 & 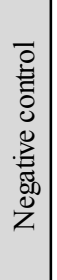 & 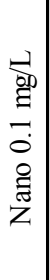 & $\mid \begin{array}{c}0 \\
z \\
. \Xi \\
\vdots \\
0 \\
\Xi \\
\vdots \\
0 \\
0 \\
\vdots\end{array}$ & 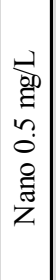 & 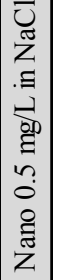 & 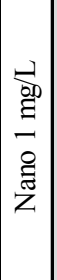 & 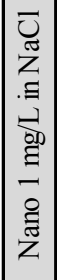 & 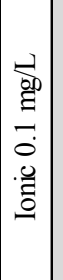 & 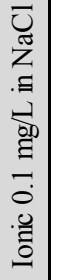 & $\mid \begin{array}{c}1 \\
0 \\
\vdots \\
n \\
0 \\
0 \\
. \\
\vdots \\
0 \\
0\end{array}$ & $\begin{array}{l}\bar{J} \\
\tilde{Z} \\
. \Xi \\
\vec{b} \\
\vdots \\
\vdots \\
0 \\
0\end{array}$ & 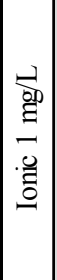 & 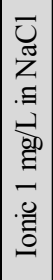 \\
\hline
\end{tabular}


Table S3. Micro/macro nutrient content in $\mathbf{1 4}$ day old $C$. annuum seedlings

\begin{tabular}{|c|c|c|c|c|c|c|c|c|c|c|c|c|c|c|c|}
\hline & 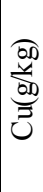 & $\mid \begin{array}{c}\tilde{\sigma} \\
\tilde{\sigma} \\
0 \\
0 \\
+1 \\
\tilde{0} \\
0 \\
0\end{array}$ & $\left|\begin{array}{c}8 \\
\tilde{\sigma} \\
8 \\
0 \\
0 \\
+1 \\
0 \\
0 \\
0 \\
0\end{array}\right|$ & $\begin{array}{l}\text {. } \\
8 \\
8 \\
0 \\
+1 \\
0 \\
0 \\
0\end{array}$ & 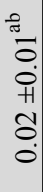 & 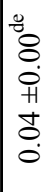 & 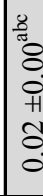 & $\delta$ & $\begin{array}{l}0 \\
0 \\
\dot{1} \\
\stackrel{1}{0} \\
0 \\
0\end{array}$ & t & $\begin{array}{l}\dot{0} \\
+ \\
\pm \\
0 \\
0\end{array}$ & 0 & & $\begin{array}{l}8 \\
\vdots \\
0 \\
+1 \\
0 \\
0 \\
\dot{0}\end{array}$ & \\
\hline & & $\mid \begin{array}{l}\sigma \\
\tilde{\sigma} \\
0 \\
0 \\
0 \\
+1 \\
0 \\
0 \\
0\end{array}$ & 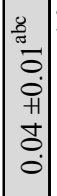 & $\begin{array}{l}\frac{\tilde{s}}{8} \\
8 \\
0 \\
0 \\
+1 \\
0 \\
0 \\
0\end{array}$ & 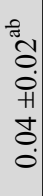 & 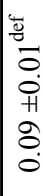 & $\begin{array}{l}\bar{O} \\
\text { Oे } \\
\text { H } \\
\text { I } \\
0\end{array}$ & $\begin{array}{l}\text { ㅍ } \\
\\
\end{array}$ & 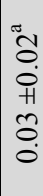 & $\stackrel{a}{a}$ & 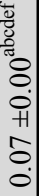 & $\begin{array}{l}+1 \\
\text { gे }\end{array}$ & $\begin{array}{l}0 \\
\text { o }\end{array}$ & $\begin{array}{l}0 \\
0 \\
0 \\
0 \\
+1 \\
0 \\
0 \\
0\end{array}$ & 0 \\
\hline & $\begin{array}{l}\frac{000}{20} \\
\frac{0}{30} \\
\frac{10}{4}\end{array}$ & $\begin{array}{l}0 \\
0 \\
0 \\
+1 \\
\hat{0} \\
0 \\
0\end{array}$ & 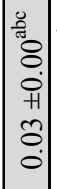 & $\begin{array}{l}8 \\
8 \\
8 \\
0 \\
+1 \\
0 \\
0 \\
0\end{array}$ & $\mid \begin{array}{l}0 \\
\vec{\sigma} \\
0 \\
0 \\
+1 \\
0 \\
0\end{array}$ & $\begin{array}{l}0 \\
0 \\
0 \\
+1 \\
0 \\
0 \\
0\end{array}$ & 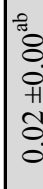 & $\begin{array}{l}8 \\
0 \\
0 \\
+1 \\
\tilde{o} \\
0\end{array}$ & 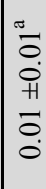 & రి. & $\begin{array}{l}8 \\
\tilde{0} \\
8 \\
0 \\
+1 \\
\\
0 \\
0\end{array}$ & $\begin{array}{l}0 \\
0 \\
0 \\
+1 \\
\mathbb{2} \\
0 \\
0\end{array}$ & 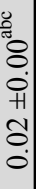 & 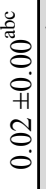 & 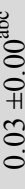 \\
\hline 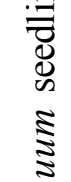 & 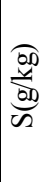 & 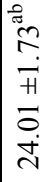 & 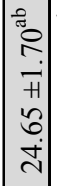 & 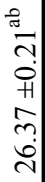 & 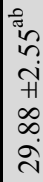 & 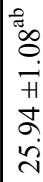 & 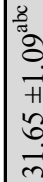 & 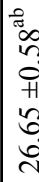 & 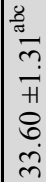 & 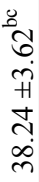 & 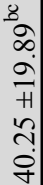 & 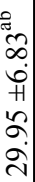 & $\begin{array}{l}\tilde{H} \\
\pm \\
\pm \\
\dot{\infty} \\
+\end{array}$ & 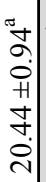 & $\bar{F}$ \\
\hline 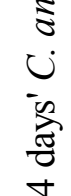 & $\begin{array}{l}\frac{500}{30} \\
\frac{50}{50} \\
\sum\end{array}$ & 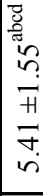 & 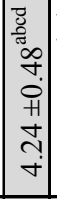 & $\begin{array}{l}\vec{z} \\
0 \\
0 \\
0 \\
+1 \\
0 \\
0 \\
0\end{array}$ & $\begin{array}{l}\stackrel{\circ}{\circ} \\
\stackrel{\circ}{H} \\
m \\
m \\
m\end{array}$ & 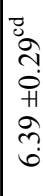 & 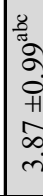 & $\begin{array}{l}\hat{y} \\
0 \\
+1 \\
\text { \& }\end{array}$ & 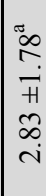 & $\begin{array}{l}J \\
\stackrel{J}{+} \\
+ \\
\infty \\
\vdots \\
\dot{v}\end{array}$ & 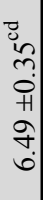 & 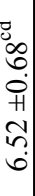 & $\stackrel{+}{+}$ & 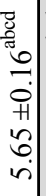 & ${ }_{\dagger}$ \\
\hline 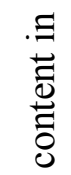 & 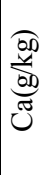 & 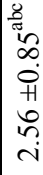 & 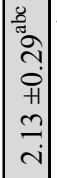 & 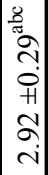 & 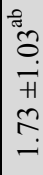 & 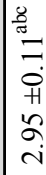 & 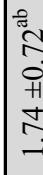 & 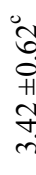 & 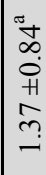 & 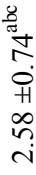 & 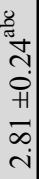 & 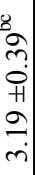 & $\begin{array}{l}\text { ㅍ } \\
\text { ते }\end{array}$ & 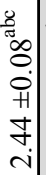 & o \\
\hline 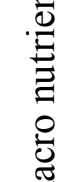 & 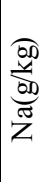 & 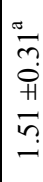 & $\left.\mid \begin{array}{l}\mathbb{1} \\
\mathfrak{n} \\
+1 \\
0 \\
0 \\
0 \\
0 \\
\mid\end{array}\right]$ & 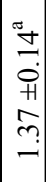 & 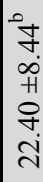 & $\mid \begin{array}{l}0 \\
0 \\
0 \\
0 \\
+1 \\
\pm \\
-1\end{array}$ & 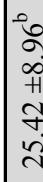 & 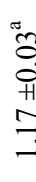 & $\mid \begin{array}{l}0 \\
0 \\
0 \\
0 \\
+1 \\
0 \\
\dot{\sim} \\
\end{array}$ & 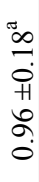 & $\begin{array}{l}0 \\
0 \\
0 \\
+1 \\
\sigma \\
i \\
m\end{array}$ & $\begin{array}{l}0 \\
? \\
0 \\
+1 \\
0 \\
\underline{ت} \\
-\end{array}$ & $\begin{array}{l}y \\
+1 \\
n \\
n \\
\infty \\
i\end{array}$ & 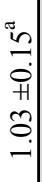 & 7 \\
\hline : & 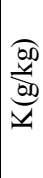 & 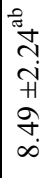 & 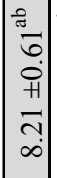 & 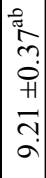 & 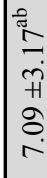 & 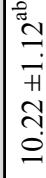 & 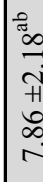 & $\begin{array}{l}8 \\
\\
+1 \\
+ \\
\infty \\
0\end{array}$ & 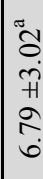 & $\begin{array}{l}\vec{J} \\
\text { H. } \\
\text { I } \\
\text {. }\end{array}$ & 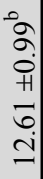 & 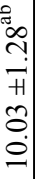 & 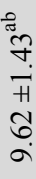 & 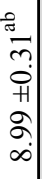 & $\approx$ \\
\hline & 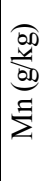 & {$\left[\begin{array}{l}\tilde{2} \\
0 \\
0 \\
1 \\
0 \\
0 \\
0\end{array}\right.$} & 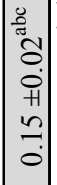 & 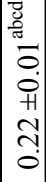 & $\begin{array}{l}\tilde{\sigma} \\
0 \\
0 \\
0 \\
= \\
0\end{array}$ & 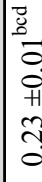 & {$\left[\begin{array}{l}\bar{w} \\
\tilde{a} \\
0 \\
0 \\
+1 \\
m \\
0 \\
0\end{array}\right.$} & $\begin{array}{l}0 \\
0 \\
\\
+ \\
c \\
2\end{array}$ & 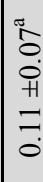 & 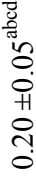 & 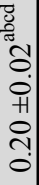 & $\begin{array}{l}0 \\
\text { n } \\
\end{array}$ & $\stackrel{0}{0}$ & 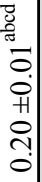 & \\
\hline & 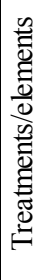 & 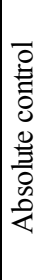 & 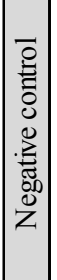 & \begin{tabular}{|c|}
0 \\
Do \\
0 \\
0 \\
0 \\
0 \\
$\vdots$ \\
\end{tabular} & 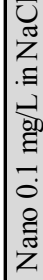 & 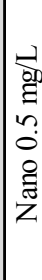 & 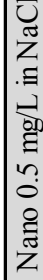 & 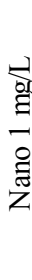 & 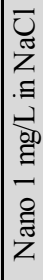 & $\begin{array}{l}\overrightarrow{b 0} \\
\underline{\Xi} \\
\overrightarrow{0} \\
.0 \\
.0\end{array}$ & 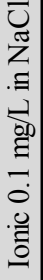 & 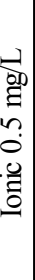 & 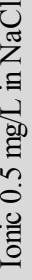 & 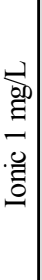 & \\
\hline
\end{tabular}




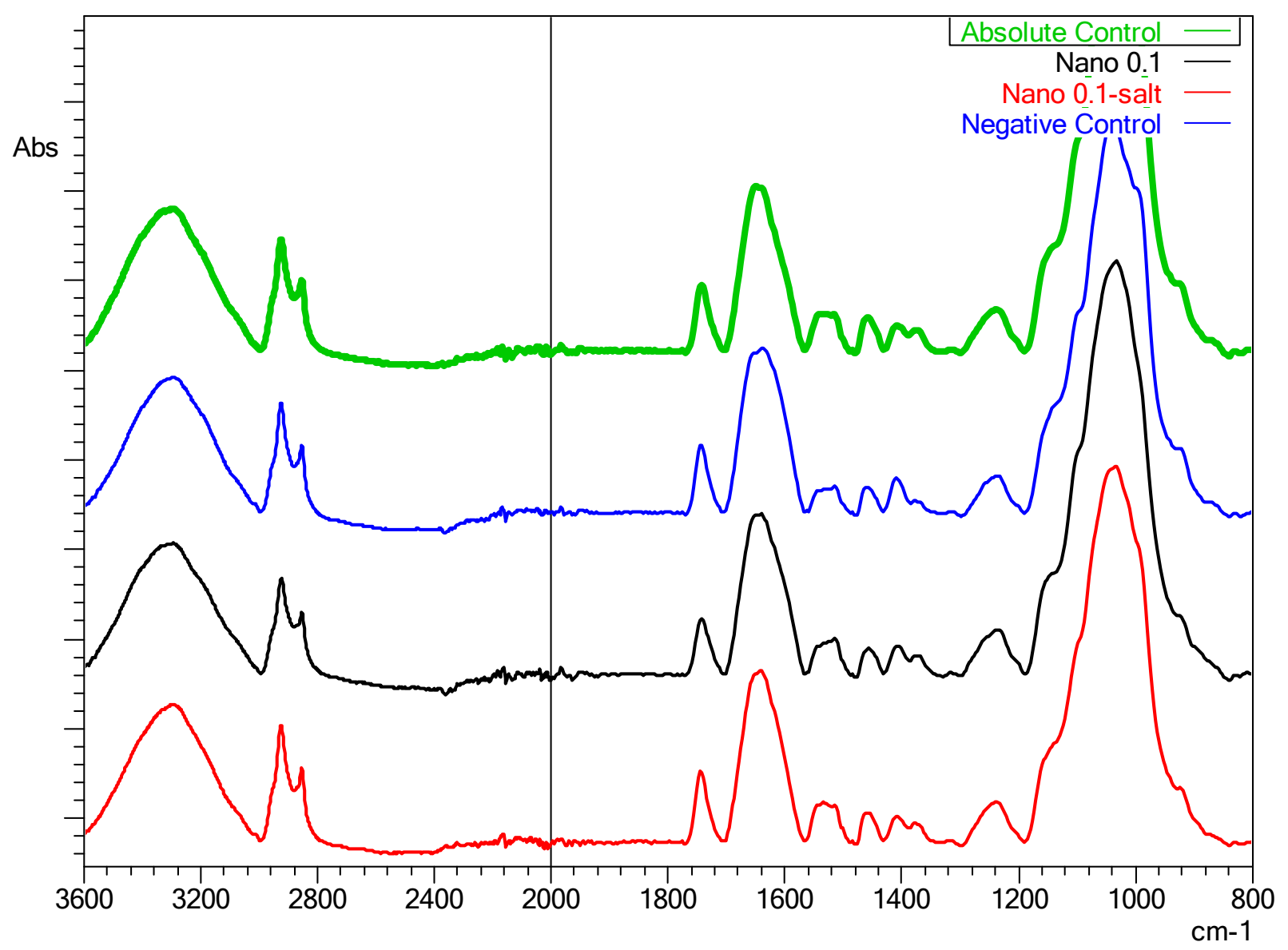

Figure S1. FTIR 


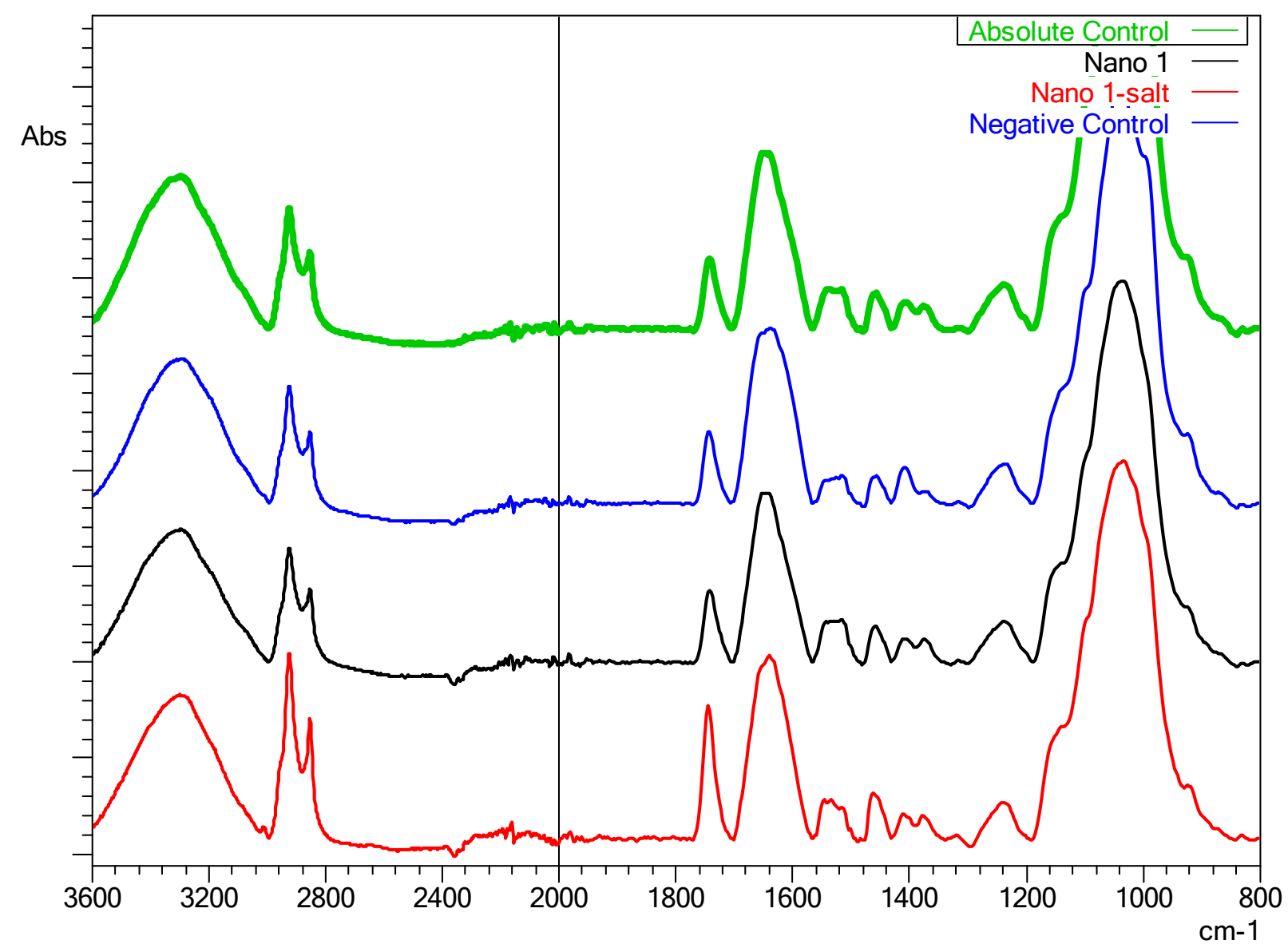

Figure S2. FTIR 


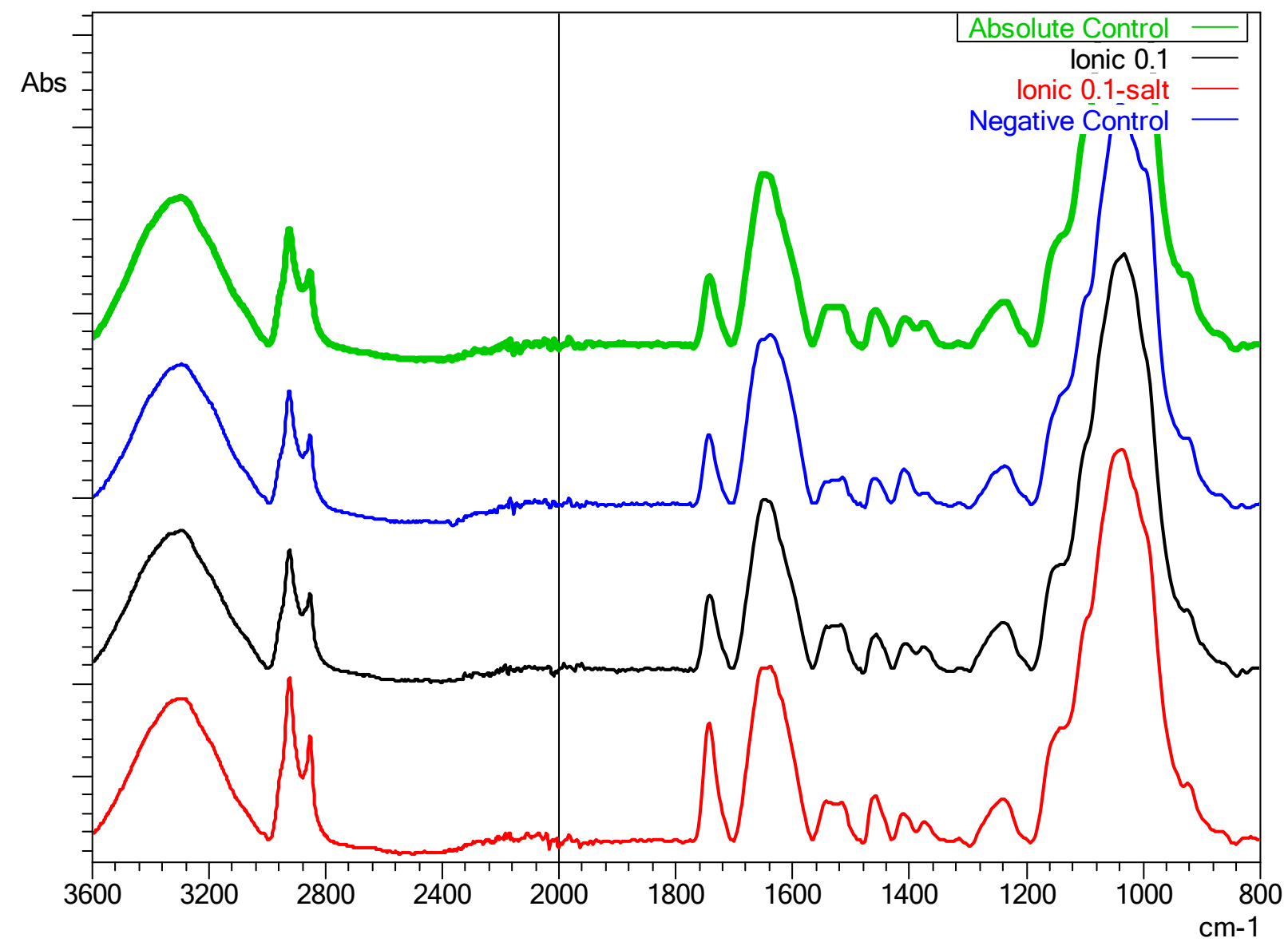

Figure S3. FTIR 


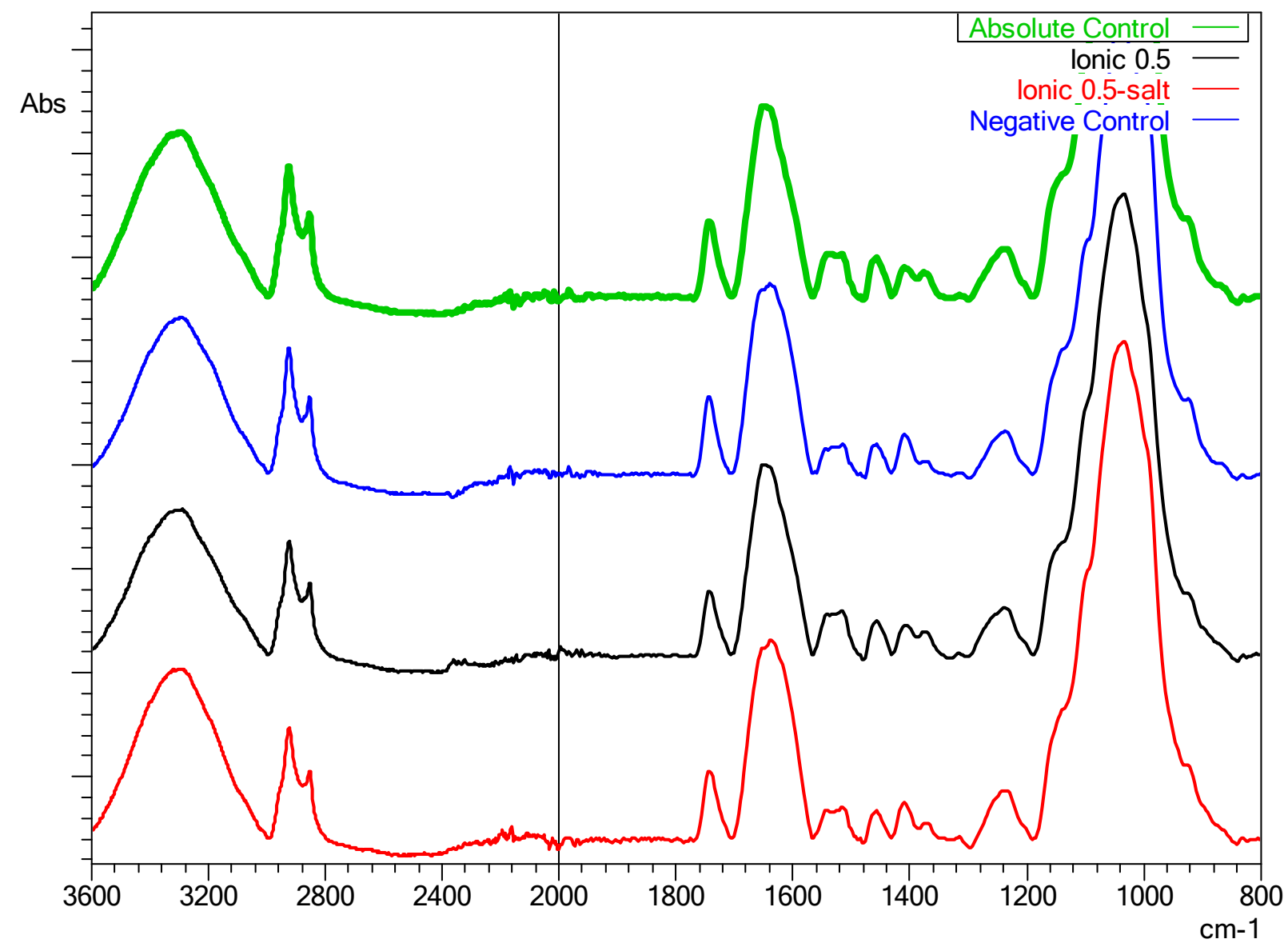

Figure S4. FTIR 


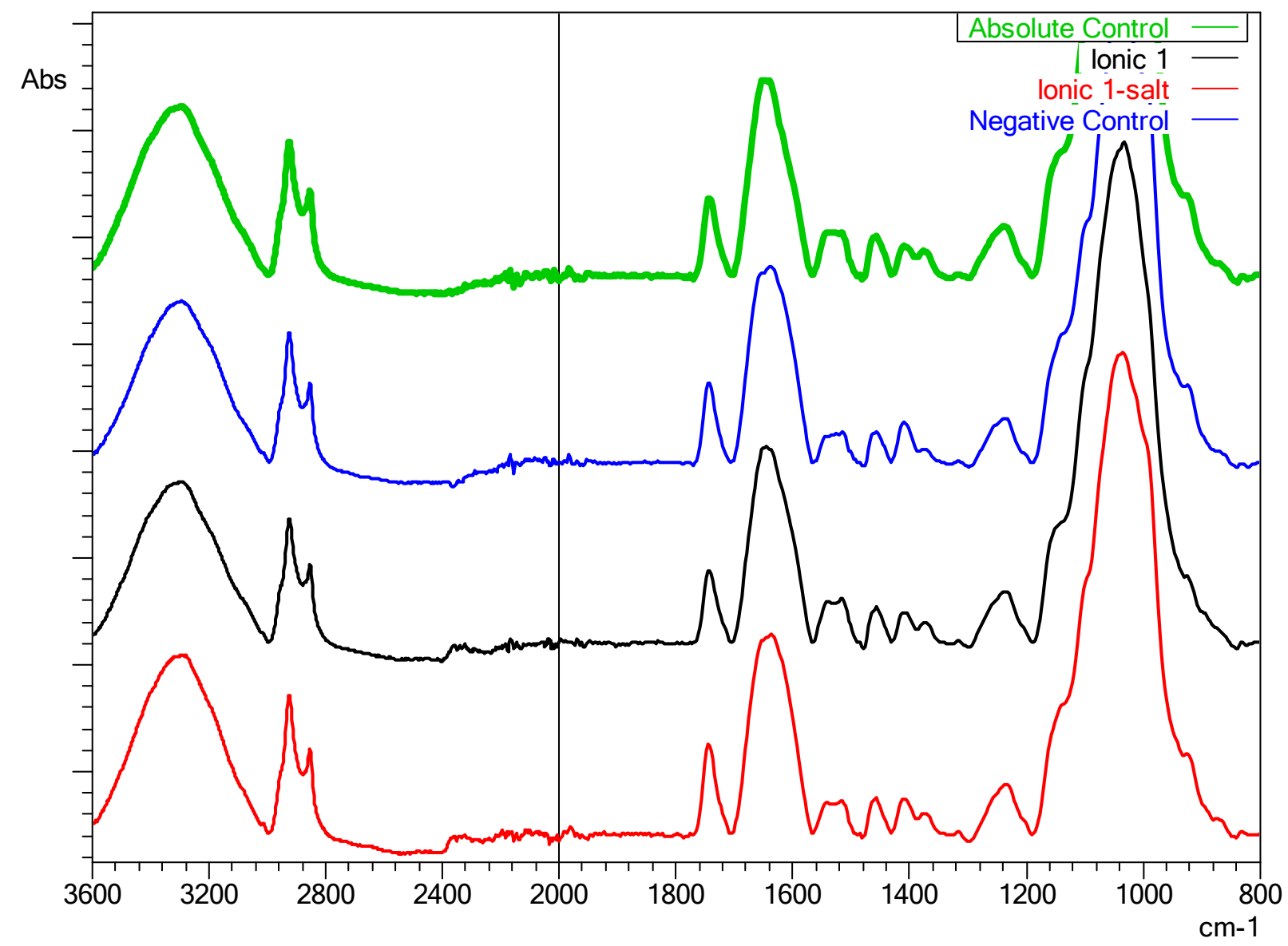

Figure S5. FTIR 
(a)

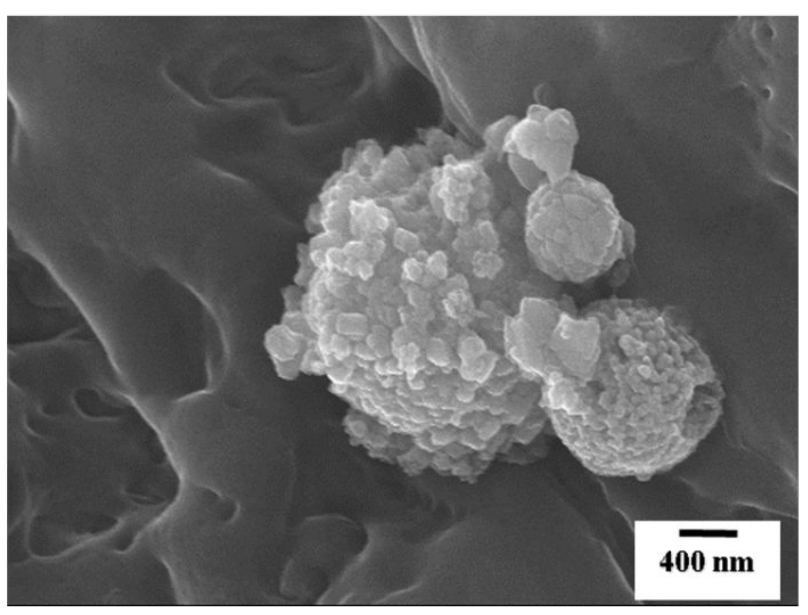

(b)

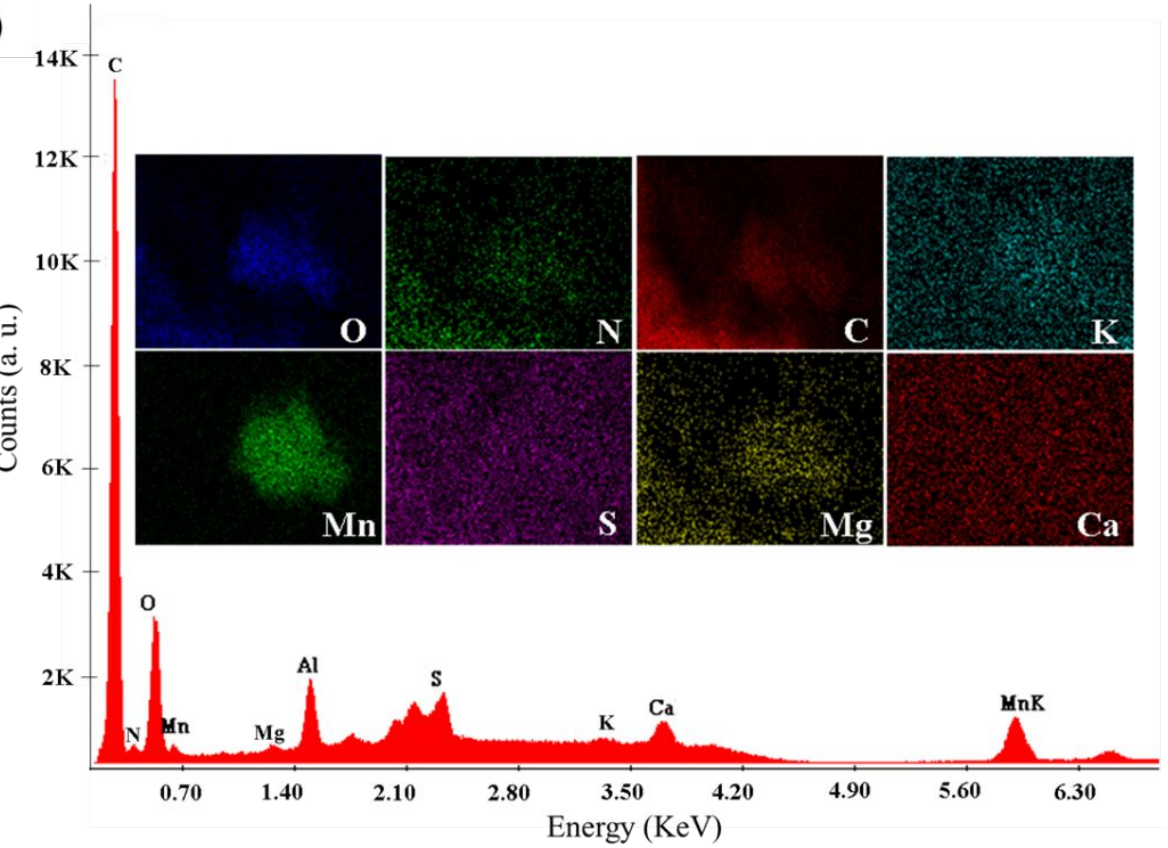

Figure S6. SEM-EDS 


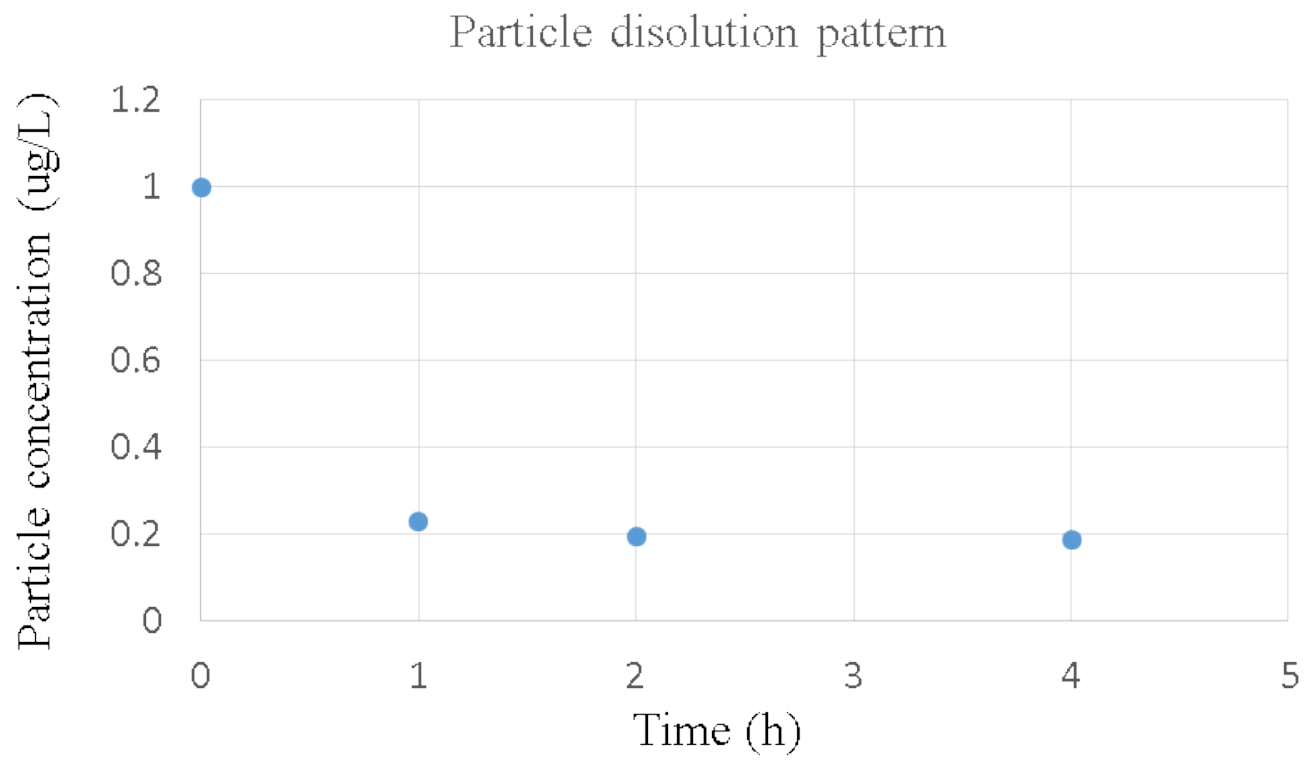

Figure S7. Dissolution study 


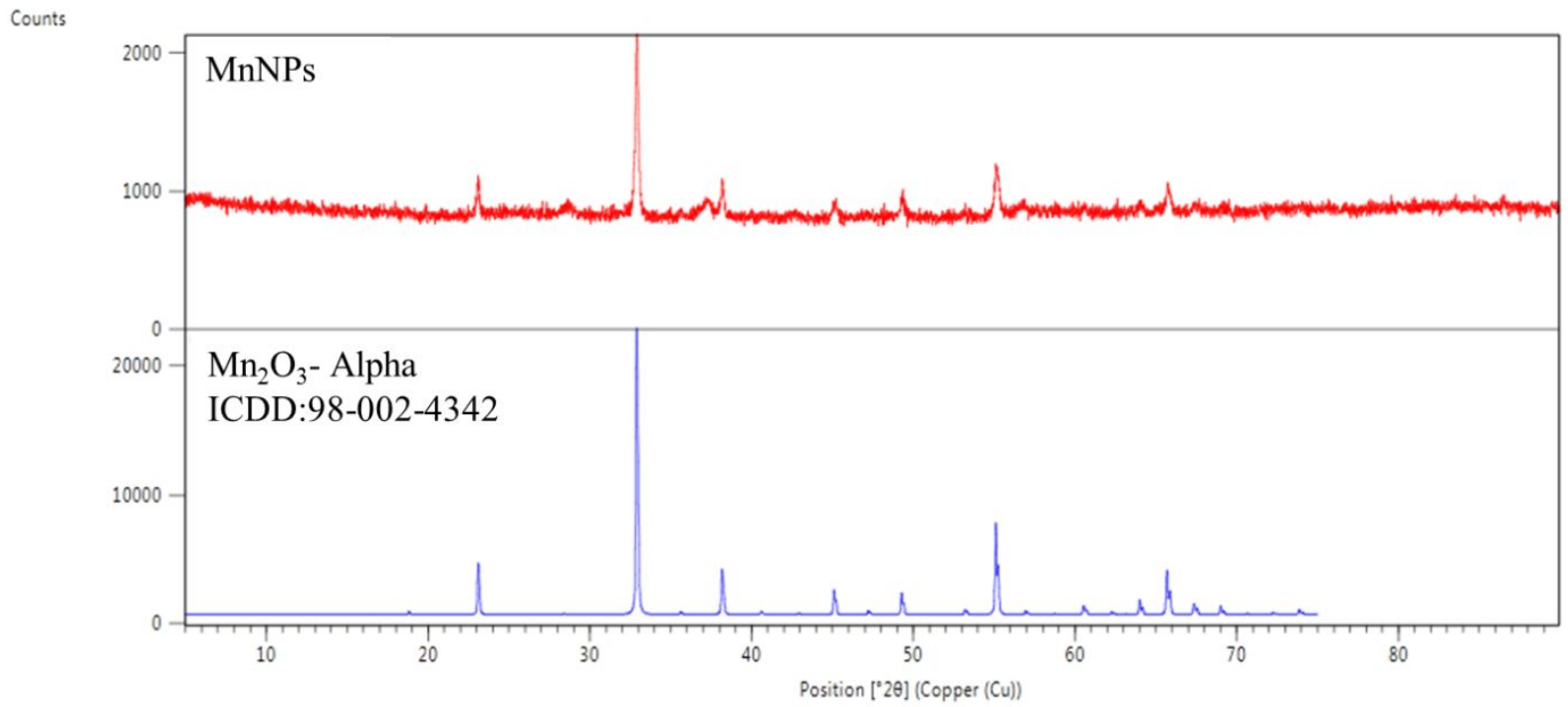

Figure S8. XRD 